\title{
Tendência da mortalidade por diabetes melito em capitais brasileiras, 1980-2007
}

\author{
Trends in mortality of diabetes mellitus patients \\ in Brazilian capitals, 1980-2007
}

Patrícia Echenique Mattos', Laércio Lima Luz², Lívia Maria Santiago²,

Inês Echenique Mattos²

\section{RESUMO}

Objetivo: Analisar o padrão de mortalidade por diabetes nas capitais brasileiras entre 1980 e 2007. Materiais e métodos: Foram calculadas taxas de mortalidade quadrienais e anuais, padronizadas por idade pela população mundial. Modelos de regressão linear foram estimados para análise da tendência nas capitais. Resultados: No primeiro quadriênio, a taxa mais elevada correspondeu a 42,89/100.000 em Aracaju e, no último, a 54,38/100.000 em São Luís. Foram observadas tendências estatisticamente significativas de incremento na maioria das capitais, embora com diferenças regionais. Belo Horizonte foi a única capital a mostrar tendência de declínio. Conclusões: Uma parte do incremento observado poderia ser atribuída a melhoras no acesso ao diagnóstico e na certificação da causa de morte, porém diferenças regionais na prevalência de fatores de risco e proteção para a doença possivelmente também estão implicadas. A não disponibilidade de série histórica de casos incidentes impossibilita determinar se esses resultados refletem tendências atuais da incidência do diabetes no Brasil. Arq Bras Endocrinol Metab. 2012;56(1):39-46

Descritores

Diabetes melito; mortalidade; tendências; capitais brasileiras

\section{ABSTRACT}

Objective: To analyze mortality patterns of patients with diabetes in Brazilian capitals from 1980 to 2007. Materials and methods: Age-standardized quadrennial and annual mortality rates were calculated with the world population as reference. Linear regression models were estimated to analyze mortality trends in each capital. Results: In the first quadrennial period, the highest rate was 42.89/100,000 in Aracaju; and in the last quadrennial period, $54.38 / 100,000$ in São Luis. Although mortality rates showed statistically significant incremental trends in most capitals, there were regional differences. Belo Horizonte was the only capital to show a declining trend. Conclusions: Part of the observed increment could be attributed to improvements in access to diagnosis and death certification, but regional differences in the prevalence of diabetes risk and protective factors might also be implicated. The absence of a time series of incident cases makes it difficult to determine if these results reflect current trends in the incidence of diabetes in Brazil. Arq Bras Endocrinol Metab. 2012;56(1):39-46

\section{Keywords}

Diabetes mellitus; mortality; trends; Brazilian capitals

\section{INTRODUÇ̃̃O}

$\mathrm{O}$ padrão epidemiológico do diabetes melito tipo 2 no mundo tem se modificado nas últimas décadas e essas mudanças têm sido atribuídas a modificações
Secretaria de Saúde do Município do Rio de Janeiro, Policlínica Rodolpho Rocco, Rio de Janeiro, RJ, Brasil 2 Departamento de Epidemiologia, Escola Nacional de Saúde Pública, Fundação Oswaldo Cruz (Fiocruz), Rio de Janeiro, RJ, Brasil
Correspondência para: Inês Echenique Mattos Escola Nacional de Saúde Pública Rua Leopoldo Bulhões, $1480,8^{\circ}$ andar, sala 817 21041-210 - Rio de Janeiro, RJ, Brasil imattos@ensp.fiocruz.br

Recebido em 23/Set/2011 Aceito em 23/Jan/2012 nos hábitos alimentares, aumento da inatividade física e da obesidade, à urbanização e ao envelhecimento da população (1). Espera-se que, entre 2000 e 2030, sua prevalência global dobre, atingindo cerca de 366 mi- 
lhões de indivíduos no mundo $(2,3)$ e diversos estudos epidemiológicos têm também demonstrado o aumento da sua incidência (4-6).

Dados do Inquérito Domiciliar sobre Fatores de Risco de Doenças Crônicas realizado pelo Ministério da Saúde revelaram que aproximadamente $40 \%$ da população brasileira de 20 anos ou mais de idade apresentava excesso de peso e $11 \%$ apresentavam obesidade (7). Indivíduos com excesso de peso possuem, com frequência, alterações derivadas da resistência à ação da insulina, associadas à distribuição central de gordura corporal, que fazem parte da chamada síndrome metabólica (8-11). Essas alterações estão associadas ao desenvolvimento do diabetes (12).

A prevalência de diabetes melito no Brasil correspondeu a 7,6\% em um estudo realizado com amostra representativa da população urbana entre 30 e 69 anos de idade (13), apresentando magnitude comparável à de países da Europa e da América do Norte $(4,6,14)$. Indivíduos com diabetes apresentam maior risco de vida, principalmente, por alterações cardiovasculares circulatórias e renais $(15,16)$. É, portanto, importante conhecer o padrão de distribuição da mortalidade por essa doença nas diferentes regiões do país.

O objetivo do presente estudo é analisar a tendência das taxas de mortalidade por diabetes na população das capitais brasileiras, entre 1980 e 2007.

\section{MATERIAIS E MÉTODOS}

Utilizou-se a série histórica de óbitos para os anos de 1980 a 2007, período em que estavam disponíveis os dados de mortalidade fornecidos pelo Sistema de Informação sobre Mortalidade (SIM) do Ministério da Saúde, com exceção de Palmas, para a qual existem informações somente a partir de 1996.

Foram selecionados no SIM todos os óbitos que ocorreram na população de estudo, cuja causa básica era o diabetes melito (códigos 250.0 a 250.9 pela CID9 e E10 a E14 pela CID-10). A população residente em cada capital no período estudado foi obtida por meio do Censo Demográfico e das estimativas populacionais estabelecidas pelo Instituto Brasileiro de Geografia e Estatística (IBGE) disponíveis no Datasus - Banco de Dados do Sistema Único de Saúde.

Efetuou-se a análise descritiva da mortalidade por diabetes melito, segundo quatro quadriênios. Para tal, foram calculadas as taxas de mortalidade quadrienais por diabetes melito para cada capital, ajustadas por ida- de pelo método direto, utilizando como padrão a população mundial.

Para análise de tendência da mortalidade foram calculadas taxas anuais de mortalidade, também ajustadas por idade, para cada capital. Foram utilizados modelos de regressão linear, sendo a variável dependente $(\mathrm{Y})$ a taxa de mortalidade de cada capital e a variável independente $(\mathrm{X})$ o ano do período de estudo. Para evitar colinearidade, a variável tempo foi centralizada mediante $\mathrm{o}$ ponto médio da série histórica.

Foram considerados como estatisticamente significativos os modelos estimados que apresentaram $\mathrm{p} \leq 0,05$.

Calculou-se, para cada capital, a mortalidade proporcional anual por causas mal definidas (códigos 780 a 799 na CID-9 e R00 a R99 na CID-10) e por diabetes, sendo comparadas ambas as distribuições e analisadas possíveis correlações por meio do coeficiente de correlação de Pearson (r), considerando como significância estatística $\mathrm{p}$ valor $\leq 0,05$.

A análise dos dados foi realizada no software SPSS for Windows versão 17.0.

\section{RESULTADOS}

$\mathrm{Na}$ tabela $\mathrm{l}$, encontram-se as taxas quadrienais de mortalidade por diabetes melito, ajustadas por idade, para as capitais das diferentes regiões brasileiras. As taxas de mortalidade mais elevadas no primeiro quadriênio foram observadas em duas capitais da região Nordeste, respectivamente 42,89/100.000 em Aracaju e 41,97/100.000 em Maceió. No último quadriênio, a região Nordeste novamente apresentou as taxas de maior magnitude, que corresponderam a $54,38 / 100.000$ em São Luís e 52,78/100.00 em Maceió. As menores taxas de mortalidade no primeiro quadriênio eram as de Cuiabá $(6,30 / 100.000)$ e Rio Branco (7,50/100.000), situadas, respectivamente, nas regiões Centro-Oeste e Norte. Já no último quadriênio, os menores valores foram observados em Florianópolis, na região Sul (16,46/100.000) e em Belo Horizonte, na região Sudeste $(18,36 / 100.000)$. A razão entre a maior e a menor taxa de mortalidade correspondeu a 6,8 e 3,3 , no primeiro e no último quadriênio, evidenciando uma redução da variabilidade da magnitude das taxas entre as regiões no período mais recente.

Pode-se observar que todas as capitais da região Norte apresentavam, nos dois primeiros quadriênios, taxas de mortalidade abaixo do valor mediano da distribuição, que correspondeu a 17,4 e 22,5/100.000. Metade das capitais dessa região apresentava taxas de mortalidade 
Tabela 1. Taxas quadrienais de mortalidade por diabetes melito (por 100.000 habitantes), ajustadas por idade, nas capitais brasileiras, 1980-2007

\begin{tabular}{lcccc}
\hline \multirow{2}{*}{ Capitais } & \multicolumn{4}{c}{ Período } \\
\cline { 2 - 5 } & $\mathbf{1 9 8 0 - 8 3}$ & $\mathbf{1 9 8 8 - 9 1}$ & $\mathbf{1 9 9 6 - 9 9}$ & $\mathbf{2 0 0 4 - 0 7}$ \\
\hline Boa Vista & 16,47 & 20,06 & 35,38 & 46,16 \\
Manaus & 9,16 & 13,78 & 21,45 & 24,89 \\
Macapá & 17,29 & 9,04 & 30,85 & 20,96 \\
Belém & 13,78 & 19,01 & 31,19 & 27,49 \\
Rio Branco & 7,50 & 17,07 & 25,52 & 48,67 \\
Porto Velho & 9,76 & 20,47 & 29,59 & 46,13 \\
Palmas* & - & - & 31,75 & 41,67 \\
São Luís & 24,75 & 30,62 & 40,02 & 54,38 \\
Teresina & 11,80 & 13,76 & 26,99 & 42,68 \\
Fortaleza & 16,37 & 18,05 & 26,81 & 21,87 \\
Natal & 16,70 & 32,24 & 38,11 & 37,54 \\
João Pessoa & 25,28 & 31,25 & 32,00 & 40,11 \\
Recife & 32,21 & 36,75 & 37,88 & 39,00 \\
Maceió & 41,97 & 43,29 & 50,26 & 52,78 \\
Aracaju & 42,89 & 34,41 & 46,47 & 50,32 \\
Salvador & 29,53 & 48,92 & 46,81 & 37,56 \\
Cuiabá & 6,30 & 17,79 & 35,24 & 38,02 \\
Campo Grande & 13,53 & 19,82 & 21,11 & 24,19 \\
Goiânia & 17,46 & 19,15 & 28,62 & 28,78 \\
Brasília & 28,79 & 28,08 & 32,14 & 32,09 \\
Belo Horizonte & 28,82 & 34,29 & 19,60 & 18,36 \\
Vitória & 31,22 & 23,95 & 40,04 & 27,38 \\
Rio de Janeiro & 39,35 & 34,76 & 38,27 & 32,36 \\
São Paulo & 28,94 & 26,81 & 29,04 & 22,05 \\
Curitiba & 19,80 & 25,41 & 26,59 & 27,07 \\
Florianópolis & 14,36 & 18,22 & 28,50 & 16,68 \\
Porto Alegre & 15,45 & 21,05 & 25,47 & 35,39 \\
\hline “ & & & \\
\hline
\end{tabular}

* Dados não disponíveis nos dois primeiros quadriênios.

que se encontravam entre os $25 \%$ menores valores no país. No terceiro quadriênio, embora a mediana tenha se elevado $(31,2 / 100.000)$, três capitais dessa região já apresentavam taxas de magnitude superior e, no último quadriênio, apresentavam, inclusive, com valores situados entre os $25 \%$ mais elevados.

As capitais da região Nordeste, em seu conjunto, apresentaram as maiores magnitudes de taxas, desde os dois primeiros quadriênios, com várias capitais registrando valores situados acima do percentil 75 da distribuição. Nos quadriênios mais recentes, mesmo com o aumento da mediana e do ponto de corte correspondente ao percentil 75, essa região ainda apresentou um grande número de capitais com taxas superiores a esses parâmetros.

A região Centro-Oeste foi aquela cujas capitais apresentaram taxas de mortalidade mais frequentemente situadas abaixo da mediana. Nos quadriênios iniciais, quase todas as capitais registraram valores situados abaixo do percentil 25 da distribuição e/ou abaixo da mediana, mantendo essas características mesmo nos anos mais recentes.

Na região Sudeste, pode-se observar um padrão de transição, com várias capitais apresentando taxas situadas acima do percentil 75 da distribuição nos primeiros quadriênios, e, no último período, registrando valores abaixo da mediana. Na região sul, observa-se que as capitais, com maiores taxas, apresentaram valores abaixo do valor mediano, ao longo do período de estudo.

Na tabela 2, são apresentados os resultados das análises de tendência da mortalidade.

$\mathrm{Na}$ região Norte, foi possível estimar modelos de regressão estatisticamente significativos para todas as capitais, com exceção de Macapá. Observou-se tendência linear de aumento das taxas de mortalidade por

Tabela 2. Tendência das taxas médias anuais ajustadas por idade de mortalidade por diabetes melito (por 100.000 habitantes) nas capitais brasileiras, 1980-2007

\begin{tabular}{|c|c|c|c|}
\hline Capital & Modelo & $\mathbf{R}^{2}$ & $p$ valor \\
\hline Boa Vista & $y=29,249+2,300 x$ & 78,3 & $<0,001$ \\
\hline Manaus & $y=16,155+0,501 x$ & 52,6 & $<0,001$ \\
\hline Macapá & $y=22,533+0,450 x$ & 10,8 & $N S^{*}$ \\
\hline Belém & $y=24,855+0,865 x$ & 60,2 & 0,001 \\
\hline Rio Branco & $y=23,449+1,761 x$ & 78,3 & $<0,001$ \\
\hline Porto Velho & $y=34,470+2,513 x$ & 72,4 & $<0,001$ \\
\hline Palmas & $y=16,434+2,049 x$ & 64,6 & $<0,001$ \\
\hline São Luís & $y=35,737+1,206 x$ & 81,6 & $<0,001$ \\
\hline Teresina & $y=24,493+1,536 x$ & 83,9 & $<0,001$ \\
\hline Fortaleza & $y=21,280+0,510 x$ & 52,2 & $<0,001$ \\
\hline Natal & $y=33,560+0,999 x$ & 37,5 & 0,001 \\
\hline João Pessoa & $y=33,348+0,744 x$ & 42,3 & $<0,001$ \\
\hline Recife & $y=35,764+0,529 x$ & 46,6 & $<0,001$ \\
\hline Maceió & $y=47,056+0,503 x$ & 29,4 & 0,004 \\
\hline Aracaju & $y=47,189+0,765 x$ & 16,0 & 0,043 \\
\hline Salvador & $y=40,155+0,447 x$ & 16,9 & 0,037 \\
\hline Cuiabá & $y=27,559+1,858 x$ & 82,0 & $<0,001$ \\
\hline Campo Grande & $y=20,404+0,653 x$ & 72,2 & $<0,001$ \\
\hline Goiânia & $y=24,136+0,749 x$ & 62,7 & $<0,001$ \\
\hline Brasília & $y=31,198+0,412 x$ & 58,7 & $<0,001$ \\
\hline Belo Horizonte & $y=25,186-0,672 x$ & 54,7 & $<0,001$ \\
\hline Vitória & $y=25,379+0,599 x$ & 38,2 & 0,001 \\
\hline Rio de Janeiro & $y=35,442+0,121 x$ & 8,6 & $N S^{*}$ \\
\hline São Paulo & $y=26,654-0,107 x$ & 12,6 & $N S^{*}$ \\
\hline Curitiba & $y=25,829+0,406 x$ & 55,7 & $<0,001$ \\
\hline Florianópolis & $y=20,366+0,399 x$ & 23,2 & 0,013 \\
\hline Porto Alegre & $y=24,949+0,755 x$ & 64,2 & $<0,001$ \\
\hline
\end{tabular}

${ }^{*}$ NS: não significativo. 
diabetes entre 1980 e 2007, com incremento médio variando entre 0,5 e 2,5 óbitos/ano.

Todas as capitais da região Nordeste apresentaram tendências de incremento da mortalidade por diabetes no período de estudo, a mesma coisa acontecendo em relação à região Centro-Oeste.

Na região Sudeste, não foi possível estimar modelo de regressão linear estatisticamente significativo para o Rio de Janeiro e para São Paulo. Nessa região, encontra-se Belo Horizonte, a única capital brasileira que apresentou tendência de declínio da mortalidade por diabetes no período de estudo, com redução média de 0,67 óbitos/ ano. Todas as capitais da região Sul apresentaram tendência de crescimento linear das taxas de mortalidade.

$\mathrm{Na}$ figura 1, pode-se visualizar a distribuição da mortalidade proporcional por causas mal definidas e por diabetes em quatro capitais que apresentaram tendência de incremento das taxas de mortalidade.

Pode-se observar que em Teresina a mortalidade proporcional por diabetes mostrou correlação forte e estatisticamente significativa com a redução da proporção dos óbitos por causas mal definidas $(r=-0,711$; $\mathrm{p}<0,001)$. Entre as capitais que evidenciaram crescimento das taxas de mortalidade, esse mesmo padrão
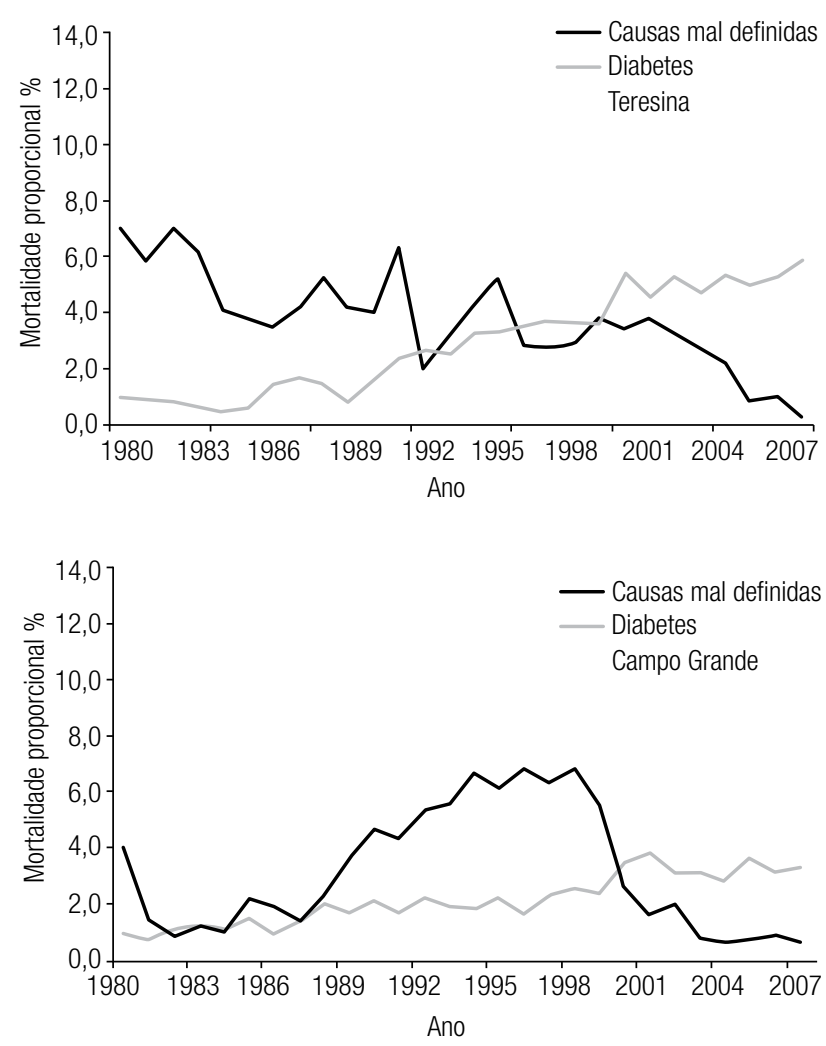

foi observado naquelas da região Norte com exceção de Manaus, em Natal, João Pessoa, Recife e Salvador no Nordeste, em todas da região Centro-Oeste, com exceção de Campo Grande e em Florianópolis no Sul. Outro padrão, exemplificado por Maceió $(\mathrm{r}=0,362 ; \mathrm{p}$ $=0,58$, que foi compartilhado por Aracaju, São Luís e Fortaleza na região Nordeste e no Norte por Manaus, caracterizou-se por um crescimento da mortalidade proporcional por diabetes, não correlacionado com as proporções anuais de óbitos por causas mal definidas. Em Campo Grande, pode-se observar que o crescimento da mortalidade proporcional por causas mal definidas não influenciou o aumento da proporção de óbitos por diabetes no período de estudo $(\mathrm{r}=-0,123 ; \mathrm{p}=0,535)$, mesmo comportamento observado para Vitória. Nessas duas cidades, as variáveis apresentaram correlações muito fracas e não estatisticamente significativas. Outro padrão foi verificado para Porto Alegre, sendo observado o crescimento da proporção de óbitos por diabetes, sem correlação com as causas mal definidas $(r=-0,042$; $\mathrm{p}=0,833$ ). Curitiba, também no Sul do país, acompanhou esse mesmo padrão. Nessas cidades, a mortalidade proporcional por causas mal definidas já apresentava patamares baixos desde a década de 1980 .
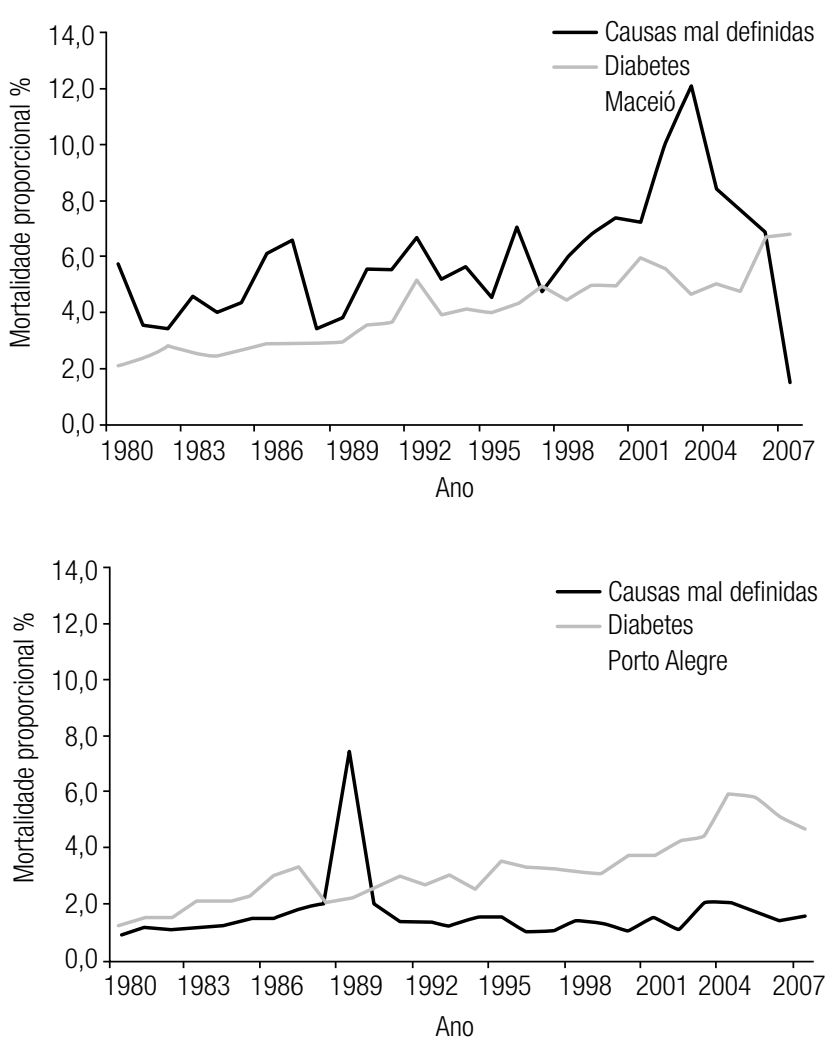

Figura 1. Mortalidade proporcional por causas mal definidas e por diabetes em quatro capitais* brasileiras.

* Capitais que apresentaram tendência de incremento das taxas de mortalidade. 


\section{DISCUSSÃO}

O aumento da prevalência de obesidade e da síndrome metabólica nos países em desenvolvimento tem sido apontado na literatura e está relacionado ao desenvolvimento de diabetes (17).

Dados da Pesquisa de Orçamento Familiar (POF) 2002-2003 permitem verificar alguns padrões de consumo alimentar negativos, que poderiam estar relacionados, pelo menos em parte, à maior prevalência de sobrepeso e obesidade que vem sendo observada no Brasil. Entre esses padrões negativos, podem ser mencionados a participação relativa da sacarose acima do recomendado ( $13,7 \%$ das calorias totais, quando se sugere que seja de até $10 \%$ ) e a baixa participação de frutas, sucos naturais, legumes, verduras e hortaliças, que, em conjunto, representaram apenas 2,3\% das calorias consumidas ao longo do dia, quando a recomendação seria de $6 \%$ a $7 \%$ (18). Além disso, foi possível observar que a prevalência de excesso de peso e de obesidade aumentava entre os homens segundo a classe de rendimento e que, entre as mulheres, aquelas que viviam com até dois salários-mínimos per capita apresentavam maior prevalência dessas duas condições (18).

No atual estágio da transição nutricional e epidemiológica da população brasileira, o diabetes melito emerge, portanto, como um importante problema de saúde pública em nosso meio. Apesar disso, as informações sistemáticas sobre a prevalência e incidência dessa doença são escassas no Brasil.

No Censo de Diabetes, realizado em nove capitais, foi encontrada prevalência ajustada por idade de 7,4\% (19). Dois estudos sobre prevalência da doença foram realizados com a população de 30 anos ou mais em cidades do estado de São Paulo, na última década. No primeiro, realizado em Ribeirão Preto, a prevalência de diabetes variou entre 3,3\% em indivíduos de 30-39 anos a $21,7 \%$ naqueles de 60-69 anos (20). Em estudo recente, também em Ribeirão Preto, foi observada prevalência de $15,0 \%$, considerando indivíduos a partir de 30 anos de idade (2l).

É conhecido que portadores de diabetes melito tipo II têm uma expectativa de vida menor e que mais de $75 \%$ deles morrem devido a complicações macrovasculares (22-24). Dessa forma, na falta de séries históricas de incidência e/ou prevalência da doença em nosso país, as informações de mortalidade ainda se constituem em um importante indicador da tendência do diabetes em nível populacional.
Neste estudo, a mortalidade por diabetes mostrou incremento na maioria das capitais brasileiras, embora diferenças regionais nas tendências das taxas tenham sido identificadas. Em estudo que analisou a tendência da mortalidade por essa doença entre 1950 e 2000, foi também observado crescimento na maioria das capitais, exceto São Paulo e Belo Horizonte, para as quais não foi possível identificar padrões estatisticamente significativos (25). Constata-se, assim, que o aumento das taxas de mortalidade por diabetes nas capitais brasileiras não é um fenômeno atual somente, mas que teve seu começo algumas décadas atrás.

Esse padrão de distribuição das taxas de mortalidade não acompanha, de modo geral, aquele evidenciado em estudos epidemiológicos realizados em diferentes países que constataram a redução da mortalidade por diabetes melito nas últimas décadas, embora com aumento da sua incidência e prevalência $(4,6,14,15)$. Entre as possíveis explicações para esse declínio, são apontadas tendências de redução da mortalidade por doença coronariana e por acidente vascular cerebral, menor exposição a fatores de risco como o tabagismo, busca ativa de casos e rastreamento levando ao diagnóstico mais precoce da doença e intervenções médicas para redução dos níveis de glicemia, colesterolemia e pressão arterial $(5,26)$.

Durante o curso do diabetes, os portadores dessa condição podem apresentar várias comorbidades e complicações que podem se refletir na mortalidade (27). Na Noruega, em estudo com pacientes internados por episódio de infarto agudo do miocárdio, foi observado que os indivíduos diabéticos apresentavam maior frequência de comorbidades como infarto prévio, angina e insuficiência cardíaca em relação aos que não apresentavam essa condição e tinham menor sobrevida (28). Comparando a mortalidade em indivíduos que apresentaram eventos coronarianos agudos, participantes de dois estudos de coorte no Reino Unido, foi verificado que, apesar dos avanços no tratamento, os ganhos de sobrevida de curto prazo observados para todo o grupo, a longo prazo, não se mantinham para os indivíduos diabéticos (23). Analisando a mortalidade por doença coronariana em indivíduos diabéticos e não diabéticos, pesquisadores da Finlândia observaram sobrevida menor entre os primeiros (29).

Parte do incremento nas taxas de mortalidade por diabetes observado neste estudo poderia ser atribuída ao maior acesso da população ao diagnóstico. Esse acesso, entretanto, possivelmente estaria ocorrendo em uma etapa mais tardia, na qual as complicações da 
doença já se instalaram e seu prognóstico é pior. Em estudo que analisou as internações da rede pública de saúde que tinham o diabetes melito como causa principal, os autores apontaram a necessidade de uma cobertura mais adequada da população, de forma a evitar a hospitalização e suas complicações (30). Outro aspecto que pode influenciar a tendência observada diz respeito a melhora da cobertura e qualidade da certificação do óbito no Brasil, que vem ocorrendo nos últimos anos (31).

Deve-se ressaltar que estimativas de mortalidade por diabetes baseadas somente na causa básica de morte podem subestimar o verdadeiro número de óbitos causados pela doença e, nesse sentido, os incrementos observados neste estudo podem ser ainda maiores. Em estudo realizado na Austrália, a principal causa de morte em diabéticos tipo II foram as doenças cardiovasculares, que responderam por $40 \%$ dos óbitos. Porém, nesse grupo de atestados de óbito, a causa básica de morte registrada foi a doença cardiovascular e não $o$ diabetes (27).

Considerando essas questões, a análise das causas múltiplas de morte, que inclui todas as causas informadas nas declarações de óbito, pode contribuir para a obtenção de um perfil mais detalhado da mortalidade por doenças crônicas (1).

São poucos os estudos brasileiros recentes que buscaram avaliar a mortalidade por diabetes com base na análise de causas múltiplas. Em estudo realizado no Rio de Janeiro, compreendendo 2.974 óbitos de indivíduos de 60 anos ou mais, o diabetes foi mencionado em $9,8 \%$ das declarações e, entre os óbitos por diabetes, $51,5 \%$ haviam sido classificados como causa básica (32). Em outro estudo (33), foi analisado o conjunto (3.872) de declarações de óbito do ano 2000 do município de Niterói, Rio de Janeiro, observando-se menção de diabetes em $313(8,1 \%)$. Após revisão e avaliação da qualidade dos dados, o diabetes foi considerado como causa básica em 154 (4,0\%), sendo obtida concordância de $95,8 \%$ na seleção da causa básica, com kappa de 0,94 . Nesse estudo, as causas associadas mencionadas com maior frequência, quando o diabetes foi considerado como causa básica, foram doenças cardiovasculares $(39,6 \%)$ e, quando o diabetes era mencionado como causa associada, a causa básica mais frequente também foi uma doença desse grupo.

Por outro lado, um grupo de pesquisadores analisou 1.315 declarações de óbito de mulheres de 10 a 49 anos, referentes ao primeiro semestre de $2002 \mathrm{em}$
26 capitais de estados brasileiros e no Distrito Federal, com o objetivo de verificar se a aplicação das Regras Internacionais de seleção da causa básica de morte possibilitava selecionar a causa básica verdadeira, mesmo quando declarada incorretamente. Os autores observaram que foi possível selecionar a verdadeira causa básica em $90,6 \%$ do total de casos, concluindo que os valores obtidos conferem alta credibilidade às estatísticas de mortalidade por causas do país (34).

A mortalidade por causas mal definidas sofreu uma importante redução em nosso país entre 1980 e 2007 (31). Dessa forma, parte das tendências de incremento observadas nas capitais poderia ser explicada pela maior qualidade da certificação das causas de óbito. De fato, foi possível observar uma correlação entre a mortalidade proporcional por causas mal definidas e por diabetes em muitas capitais, em especial das regiões Norte e Nordeste, que poderiam ser parcialmente explicadas por essa redução. Entretanto, em outras capitais que também apresentaram tendências de crescimento das taxas de mortalidade por diabetes, não foram observadas correlações entre essas variáveis, indicando que esse incremento possa estar relacionado ao aumento da incidência da doença na população.

As variações observadas em relação à tendência da mortalidade por diabetes melito nas capitais brasileiras apontam para a necessidade de se avaliar o papel de indicadores de acesso e utilização dos serviços, além de características populacionais específicas, como possíveis explicações. Diferenças regionais na prevalência de fatores de risco para o diabetes e/ou na qualidade da atenção de saúde recebida pelos indivíduos com a doença possivelmente explicam uma parcela desses resultados.

No estudo das internações por diabetes na rede pública de saúde do Brasil, os autores observaram variações regionais na razão número de hospitalizações por habitantes (30). No Canadá, a mortalidade de indivíduos diabéticos no período 1994-2005 foi analisada com base em categorias de renda, sendo verificada redução estatisticamente significativa, no grupo da população que apresentava rendimento mais alto em comparação com aquele de menor renda (16). Nos Estados Unidos, foi observado que os progressos alcançados no cuidado do diabetes trouxeram maior benefício para os indivíduos com alta escolaridade (35).

Este estudo apresenta limitações, entre elas, a possibilidade de que os dados de mortalidade estejam subestimados, por se ter trabalhado somente com a causa 
básica de morte, sem considerar as demais causas informadas nas declarações de óbito. No caso do diabetes, particularmente, essa causa costuma aparecer com frequência como associada, principalmente em populações idosas, nas quais estão presentes outras comorbidades $(25,34)$. Por outro lado, em uma análise das relações entre diabetes e doenças cardiovasculares como causas associadas de morte nas declarações de óbito, foi apontado que, em anos mais recentes, tem sido observada em vários países maior tendência de colocá-lo na parte I do atestado, o que levaria a possível superestimação da mortalidade pela doença $(1)$.

Em decorrência da não disponibilidade de séries históricas de incidência/prevalência da doença, que possibilitem a realização de comparações, não se tem como identificar se os achados deste estudo estão refletindo tendências reais na incidência de diabetes no Brasil e/ou questões ligadas à qualidade da atenção à saúde recebida pelos indivíduos doentes influenciando diretamente a mortalidade. Entretanto, a relevância de intervenções voltadas para modificações no estilo de vida da população como medidas efetivas na prevenção tanto do diabetes melito como de suas complicações é consenso na literatura $(10,17,21,36)$.

Considerando o crescimento importante da mortalidade por diabetes melito que vem sendo observado na maioria das capitais brasileiras, é necessário que essas questões estejam no cerne das políticas públicas de prevenção e de promoção da saúde desenvolvidas no país.

Declaração: os autores declaram não haver conflitos de interesse científico neste estudo.

\section{REFERÊNCIAS}

1. AdairT, Rao C. Changes in certificates of diabetes with cardiovascular diseases increased reported diabetes mortality in Australia and the United States. J Clin Epidemiol. 2010;63:199-204.

2. Jansson SPO, Anderssdon DKG, Svärdsudd K. Prevalence and incidence rate of diabetes mellitus in a Swedish community during 30 years of follow-up. Diabetol. 2007;50:703-10.

3. Chan JCN, Vasanti M, Weiping J, Kadowaski T, Yajnik CS, Yoon KH, et al. Diabetes in Asia: epidemiology, risk factors and pathophysiology. J Am Med Assoc. 2009;301(20):2129-40.

4. Lipscombe LL, Hux JE. Trends in diabetes prevalence, incidence and mortality in Ontario, Canada 1995-2005: a population-based study. Lancet. 2007;369:750-6.

5. Charlton J, Latinovic R, Gulliford MC. Explaining the decline in early mortality in men and women with type 2 diabetes: population-based cohort study. Diab Care. 2008;31(9):1761-6.

6. Carstensen B, Kristensen JK, Ottosen P, Borch-Johnsen K. The Danish National Diabetes Register: trends in incidence, prevalence and mortality. Diabetol. 2008;51:2187-96.
7. BRASIL/Ministério da Saúde. Inquérito domiciliar sobre comportamentos de risco e morbidade referida de doenças e agravos não transmissíveis: Brasil, 15 capitais e Distrito Federal, 20022003. Rio de Janeiro: Inca, 2004.

8. Zimmet P, Magliano D, MatsuzawaY, Alberti G, Shaw J. The metabolic syndrome: a global public health problem and a new definition. J Atheros Thromb. 2005;12(6):295-300.

9. Sorrentino MJ. Implications of the metabolic syndrome: the new epidemic. Am J Cardiol. 2005;96(suppl):3E-7E.

10. Foreyt JP. Need for lifestyle intervention: how to begin. Am J Cardiol. 2005;96:4A.

11. Vitarius JA. The metabolic syndrome and cardiovascular disease. Mt Sinai J Med. 2005;72(4):257-62.

12. Ford ES, Schulze MB, PischonT, Bergmann MM, Joost HG, Boeing $\mathrm{H}$. Metabolic syndrome and risk of incident diabetes: findings from the European Prospective Investigation into cancer and nutrition - Postdam Study. Cardiovasc Diabetol. 2008;7:35.

13. Bosi PL, Carvalho AM, Contrera D, Casale G, Pereira MA, Gronner $M F$, et al. Prevalência de diabetes melito e tolerância à glicose diminuída na população urbana de 30 a 79 anos da cidade de São CarIos, São Paulo. Arq Bras Endocrinol Metabol. 2009;53(6):726-32.

14. Evans JMM, Barnett KN, Ogston SA, Morris AD. Increasing prevalence of type 2 diabetes in a Scottish population: effect of increasing incidence or decreasing mortality? Diabetol. 2007;50:729-32.

15. Eliasson $M$, Talbäck $M$, Rosén $M$. Improved survival in both men and women with diabetes between 1980 and 2004 - a cohort study in Sweden. Cardiovasc Diabetol. 2008;7:32.

16. Lipscombe LL, Austin PC, Manuel DG, Shah BR, Hux JE, Booth $\mathrm{GL}$. Income-related differences in mortality among people with Diabetes mellitus. Canad Med Assoc J. 2010;182(1):E1-E16.

17. Misra A, Khurana L. Obesity and the metabolic syndrome in developing countries. J Clin Endocrinol Metab. 2008;93(11):S9-S30.

18. BRASIL/Instituto Brasileiro de Geografia e Estatística. Pesquisa de Orçamento Familiar (POF) 2002-2003. Disponível em: http:// www.ibge.gov.br/home/estatistica/populacao/condicaodevida/ pof/2002analise/ Acesso em: Jul 2010.

19. Malerbi DA, Franco LJ. Multicenter study of the prevalence of diabetes mellitus and impaired glucose tolerance in the urban Brazilian population aged 30-69 yr. The Brazilian Cooperative Group on the Study of Diabetes Prevalence. Diab Care. 1992;15:1509-16.

20. Torquato MTCG, Montenegro Jr RM, Viana LAL, Souza RAHG, Lanna CMM, Lucas JCB, et al. Prevalence of diabetes mellitus and impaired glucose tolerance in the urban population aged 30-69 years in Ribeirão Preto (São Paulo), Brazil. S Paulo Med J. 2003;12(6):224-30.

21. Moraes SA, Freitas ICM, Gimeno SGA, Mondini L. Prevalência de diabetes mellitus e identificação de fatores associados em adultos residentes em área urbana de Ribeirão Preto, São Paulo, Brasil, 2006: Projeto OBEDIARP. Cad S Pub. 2010;26(5):929-41.

22. Davies MJ, Tringham JR, Troughton J, Kunti KK. Prevention of type II diabetes mellitus; a review of the evidence and its application in a UK setting. Diab Med. 2004;21:403-14.

23. Cubbon RM, Wheatcroft SB, Grant PJ, Gale CP, Barth JH, Sapsford RJ, et al. Temporal trends in mortality of patients with diabetes mellitus suffering acute myocardial infarctation: a comparison of over 3000 patients between 1995 and 2003. Eur Heart J. 2007;28:540-5.

24. Preis SR, Hwang SJ, Coady S, Pencina MJ, D'Agostino RB, Savage P, et al.Trends in all-cause and cardiovascular disease mortality among women and men with and without diabetes mellitus in the Framingham Heart Study, 1950 to 2005. Circulation. 2009;119:1728-35.

25. Cesse EAP, Carvalho EF, Souza WV, Luna CF. Tendência da mortalidade por diabetes melito no Brasil: 1950 a 2000. Arq Bras Endocrinol Metabol. 2009;53(6):760-6. 
26. Gulliford $M C$, Charlton J. Is relative mortality of type 2 diabetes mellitus decreasing? Am J Epidemiol. 2009;169(4):455-61.

27. Bi P, Parton K, Donald K. Secular trends in mortality rates for diabetes in Australia. Diab Res Clin Practice. 2005;70:270-7.

28. Kvan E, Pettersen KI, Sandvik L, Reikvam A. High mortality in diabetic patients with acute myocardial infarction: cardiovascular co-morbidities contribute most to the high risk. Int J Cardiol. 2007;121:184-8.

29. Forssas EH, Keskimäki IT, Reunannen AR, Koskinen SV. Coronary heart disease among diabetic and nondiabetic people - socioeconomic differences in incidence, prognosis and mortality. J Diab Complic. 2008;22:10-7.

30. Rosa RS, Schmidt MI, Duncan BB, Souza MFM, Lima AK, Moura L. Internações por diabetes mellitus como diagnóstico principal na rede pública do Brasil, 1999-2001. Rev Bras Epidemiol. 2007;10(4):465-78.

31. BRASIL/Instituto Brasileiro de Geografia e Estatística. A qualidade da informação sobre a mortalidade no Brasil recente e avaliação do impacto das causas violentas no número de anos de vida perdidos. Disponível em: http://www.ibge.gov.br/home/esta- tistica/populacao/indic_sociosaude/2009/com_aquali.pdf. Acesso em: Ago 2011.

32. Coeli CM, Ferreira LGFD, Drbal MM, Veras RP, Camargo Jr KR, Cascão AM. Mortalidade em idosos por diabetes mellitus como causa básica e associada. Rev Saúde Pública. 2002;36(2):135-40.

33. Maya LG. Confiabilidade do diabetes mellitus referido como causa de morte: análise comparativa da mortalidade segundo método de causas múltiplas, Niterói (RJ), 1993 e 2000. Dissertação de Mestrado, Escola Nacional de Saúde Pública. 2003.

34. Laurenti R, Mello Jorge MHP, Gotlieb SLD. Informação em mortalidade: o uso das regras internacionais para a seleção da causa básica. Rev Bras Epidemiol. 2009;12(2):195-203.

35. Miech RA, Kim J, McConnell C, Hamman RF. A growing disparity in diabetes-related mortality US trends, 1989-2005. Am J Prev Med. 2009;36(2):126-32.

36. WHO - World Health Organization. Diet, nutrition and the prevention of chronic diseases. Report of a Joint WHO/FAO Expert Consultation. WHOTechnical Report Series, 916. Geneva, 2003. 